\section{Portfolio Optimization with Markov-modulated stock prices and interest rates}

\author{
Nicole Bäuerle and Ulrich Rieder
}

\begin{abstract}
A financial market with one bond and one stock is considered where the risk free interest rate, the appreciation rate of the stock and the volatility of the stock depend on an external finite state Markov chain. We investigate the problem of maximizing the expected utility from terminal wealth and solve it by stochastic control methods for different utility functions. Due to explicit solutions it is possible to compare the value function of the problem to one where we have constant (average) market data. The case of benchmark optimization is also considered.
\end{abstract}

Index Terms-Markov-modulation, stochastic control, verification theorem, utility maximization

\section{INTRODUCTION}

The financial market considered in this paper is incomplete and consists of one bond and one risky asset. The incompleteness of the market is due to stochastic coefficients appearing in the price process of the risky asset and the bond. More precisely we assume that the interest rate of the bank account, the appreciation rate of the stock and the volatility of the stock depend on an external continuoustime, finite state Markov chain $Y$. The state of the Markov chain should represent the general market conditions (for a motivation see e.g. [23]). Models with deterministic coefficients are only good for a relative short period of time and cannot respond to changing conditions. In this Markov-modulated setting we want to solve the classical portfolio optimization problem where an investor wants to maximize the expected utility from terminal wealth. As far as the information is concerned, the investor has at the time point of decision, we show that it makes no difference whether we assume that the agent can only observe the stock price process or whether he can observe the stock price and the market condition $Y$. This is due to the fact that in a diffusion price process model the quadratic variation and thus the volatility can be approximated arbitrarily well by the price process (cf. [9]). Therefore it is in principle sufficient to solve the optimization problem with complete observation. This is done using stochastic control methods for a number of different utility functions, namely for logarithmic utility, CRRA utility and for benchmark optimization.

Motivated by the paper of Merton in 1971, there is a growing literature dealing with portfolio optimization problems under different aspects. Problems with stochastic volatility have for example been investigated in [4], [21], [22] and [8] among others. Most of these papers assume that the external process is a diffusion process itself, like in the established volatility model of Heston (1993). To the best of our knowledge the first paper to model the volatility as a continuous-time Markov chain is [3]. As we will see this model has the advantage that many portfolio problems can be solved explicitly in contrast to the diffusion setting (compare for example [4], [8]). Moreover, a diffusion process can be approximated arbitrarily closely by a continuous-time Markov chain (see [16]). Portfolio optimization with stochastic interest rates are e.g. treated in [17] and [14]. The authors of [14] consider the Ho-Lee and the Vasicek model for the interest rate which are both diffusion processes.

The solutions we obtain are found with the help of stochastic control methods. More precisely by the use of a verification theorem. For a comprehensive presentation of this theory the reader is referred to [5] or [6] among others.

Our paper is organized as follows: in section II we present a precise mathematical framework for our model and we shortly comment on the case with incomplete information In section II-A we prove a verification theorem which is useful for our portfolio problems. In section II-B, and II-C we solve the investment problems with logarithmic utility and CRRA utility. In these cases it turns out that it is optimal to invest a constant fraction of the wealth in the stock, depending on the current market condition $Y$. The value function of these portfolio problems can be written in such a way that it is easy to compare the stochastic coefficient problem to one where we have constant (average) coefficients. The result of the comparison depends on the utility function. Finally we investigate in section IID the benchmark optimization problem. In the case of deterministic coefficients this model has been considered in [1] and in a more general context by the paper [7]. In our setting we are only partly able to solve the portfolio problem explicitly. A closed form solution is derived when the discounted stock price process is a martingale. In the general model some statements concerning asymptotic optimality are shown.

\section{THE MOdeL}

We consider a financial market with one bond and one risky asset. More precisely let $\left(\Omega, \mathcal{F}, \mathfrak{F}=\left\{\mathcal{F}_{t}, 0 \leq t \leq T\right\}, P\right)$ be a filtered probability space. We assume that $\mathcal{F}=\mathcal{F}_{T} . T>0$ is a fixed time horizon. The bond price process $B=\left(B_{t}\right)$ evolves according to

$$
d B_{t}=r\left(Y_{t}\right) B_{t} d t
$$

and the stock price process $S=\left(S_{t}\right)$ according to

$$
d S_{t}=\mu\left(Y_{t}\right) S_{t} d t+\sigma\left(Y_{t}\right) S_{t} d W_{t}
$$

where $W=\left(W_{t}\right)$ is a Brownian motion and $Y=\left(Y_{t}\right)$ is a continuous-time Markov chain with finite state space $E$ and intensity matrix $Q=\left(q_{i j}\right)_{i, j \in E}$ under $P$ w.r.t. $\mathfrak{F}$. $W$ and $Y$ are assumed to be independent. In what follows we suppose that $Y$ is càglàd and that $\mu, r, \sigma: E \rightarrow \mathbb{R}_{+}$and $\mu(i)>r(i)>0$ for all $i \in E$.

Our model allows for random jumps of the interest rate $r$, the appreciation rate $\mu$ and volatility $\sigma$. These jumps can be due to changes of external economic factors. Of course in most of the established models the volatility or the interest rate is given by a diffusion process, like for example in the Heston model (1993), the Ho-Lee model (1986) or the Vasicek model (1977). However, in our case the portfolio optimization problem is simpler to solve (compare e.g. [8], [4]) and it is well-known that diffusion processes can be approximated arbitrarily well by continuous-time Markov chains (see e.g.[16]). Therefore, these standard models can be approximated by our jump model.

The optimization problem is to find investment strategies that maximize the expected utility from terminal wealth. In what follows we denote by $\pi_{t}$ the fraction of wealth invested in the stock at time $t$. The process $\pi=\left(\pi_{t}\right)$ is called portfolio strategy. A portfolio strategy is admissible, whenever $\int_{0}^{T} \pi_{s}^{2} d s<\infty$ almost surely. By $X^{\pi}=\left(X_{t}^{\pi}\right)$ we denote the corresponding wealth process. The selffinancing assumption gives us the following stochastic differential equation for the wealth process

$$
\begin{aligned}
d X_{t}^{\pi} & =X_{t}^{\pi} \pi_{t} \frac{d S_{t}}{S_{t}}+X_{t}^{\pi}\left(1-\pi_{t}\right) \frac{d B_{t}}{B_{t}} \\
& \left.=X_{t}^{\pi}\left[r\left(Y_{t}\right)+\pi_{t}\left(\mu\left(Y_{t}\right)-r\left(Y_{t}\right)\right) d t\right]+X_{t}^{\pi} \pi_{t} \sigma\left(Y_{t}\right) d W_{t}\right](3)
\end{aligned}
$$

with $X_{0}^{\pi}=x>0$ being the initial wealth. This linear stochastic differential equation can be solved explicitly and the solution is given by

$$
\begin{aligned}
X_{t}^{\pi}= & x \cdot \exp \left\{\int_{0}^{t} r\left(Y_{s}\right)+\pi_{s}\left(\mu\left(Y_{s}\right)-r\left(Y_{s}\right)\right)-\frac{1}{2} \sigma\left(Y_{s}\right)^{2} \pi_{s}^{2} d s\right. \\
& \left.+\int_{0}^{t} \sigma\left(Y_{s}\right) \pi_{s} d W_{s}\right\} .
\end{aligned}
$$


Finally we are given a concave and increasing utility function $U$ : $(0, \infty) \rightarrow \mathbb{R}$. Our aim is to solve the investment problem

$$
\sup E^{x}\left[U\left(X_{T}^{\pi}\right)\right]
$$

where the supremum is taken over all admissible portfolio strategies and $E^{x}$ is the conditional expectation, given $X_{0}^{\pi}=x$.

A crucial question always concerns the information which is available at decision time points. It seems to be natural to consider a market where the agents are able to observe the stock price process $S$ only. This situation is referred to as partial observation. The case of complete information is given, when agents can observe the Brownian motion $W$ as well as the driving process $Y$ for the market data. However, the stock price process contains enough information to filter the evolution of $W$ and $Y$ from it. This is due to the fact that the quadratic variation of the stock price process $<S, S>_{t}=\int_{0}^{t} S_{\tau}^{2} \sigma^{2}\left(Y_{\tau}\right) d \tau$, can be observed and thus in case $\sigma(\cdot)$ is bijective, also $Y$ (for more details the reader is referred to Pham/Quenez (2001)). Hence, the case of partial observation can be reduced to the case of complete information and we assume now that the agent knows upon the time point $t$ the evolution of $W$ and $Y$ until time $t$. Or, what is equivalent, at time $t$ he knows his current wealth and the state of $Y_{t}$. Hence we assume that the filtration $\mathfrak{F}$ is generated by $W$ and $Y$, i.e. $\mathcal{F}_{t}=\sigma\left(W_{s}, Y_{s}, s \leq t\right)$. The set of admissible strategies over the planning period $[t, T]$ is given by

$$
\begin{aligned}
\mathcal{A}(t, \mathfrak{F}):= & \{\pi:[t, T] \rightarrow \mathbb{R} \mid \pi \text { is } \mathfrak{F} \text { - adapted }, \\
& \left.\int_{t}^{T} \pi_{s}^{2} d s<\infty \text { a.s. }\right\} .
\end{aligned}
$$

Note that $\pi_{t}$ is not restricted to the interval $[0,1] . \pi_{t}<0$ means that the stock is sold short and $\pi_{t}>1$ means that money is borrowed from the bank at the interest rate $r\left(Y_{t}\right)$. By $E^{t, x, i}$ we denote the conditional expectation, given $X_{t}=x$ and $Y_{t}=i$. The optimization problem is now

$$
\sup _{\pi \in \mathcal{A}(0, \mathfrak{F})} E^{0, x, i}\left[U\left(X_{T}^{\pi}\right)\right] .
$$

We are going to solve this problem via stochastic control. As usual it is convenient to denote by

$$
V(t, x, i):=\sup _{\pi \in \mathcal{A}(t, \mathfrak{F})} E^{t, x, i}\left[U\left(X_{T}^{\pi}\right)\right]
$$

the value function of the investment problem over time horizon $[t, T]$. The key equation to solve the problem is the so-called HamiltonJacobi-Bellman (HJB) equation. It reads in this case

$$
\begin{gathered}
\sup _{u \in \mathbb{R}}\left\{V_{t}+x[r(i)+u(\mu(i)-r(i))] V_{x}+\frac{1}{2} x^{2} u^{2} \sigma^{2}(i) V_{x x}\right. \\
\left.+\sum_{j \in E} q_{i j}[V(t, x, j)-V(t, x, i)]\right\}=0
\end{gathered}
$$

with the boundary condition $V(T, x, i)=U(x)$. In what follows we will abbreviate the term appearing in brackets by $\mathcal{G}^{u} V(t, x, i)$. In the next section we will see how to obtain a solution of the optimization problem with the help of the HJB equation.

Remark 1: In principle we could restrict to the case where only the interest rate and the volatility depends on $Y$. There is no extra generality in allowing the appreciation rate to depend on $Y$ also. This can be seen as follows: suppose $\left(\pi_{t}^{*}\right)$ is the optimal strategy for the portfolio problem with wealth process $\left(X_{t}^{\pi}\right)$, given by

$$
d X_{t}^{\pi}=X_{t}^{\pi}\left[r\left(Y_{t}\right)+\pi_{t}\left(\tilde{\mu}-r\left(Y_{t}\right)\right)\right] d t+X_{t}^{\pi} \pi_{t} \tilde{\sigma}\left(Y_{t}\right) d W_{t}
$$

where $\tilde{\sigma}(i)=\frac{\tilde{\mu}-r(i)}{\mu(i)-r(i)} \sigma(i)$. Then $\pi^{* *}=\left(\pi_{t}^{* *}\right)$ with

$$
\pi_{t}^{* *}=\frac{\tilde{\mu}-r\left(Y_{t}\right)}{\mu\left(Y_{t}\right)-r\left(Y_{t}\right)} \pi_{t}^{*}
$$

is obviously optimal for the portfolio problem with wealth process given by

$$
d X_{t}^{\pi}=X_{t}^{\pi}\left[r\left(Y_{t}\right)+\pi_{t}\left(\mu\left(Y_{t}\right)-r\left(Y_{t}\right)\right)\right] d t+X_{t}^{\pi} \pi_{t} \sigma\left(Y_{t}\right) d W_{t} .
$$

\section{A. A Verification Theorem}

In our model a solution of the HJB-equation (4) gives us indeed the optimal value function $V(t, x, i)$ and the optimal portfolio strategy $\pi^{*}=\left(\pi_{t}^{*}\right)$. This is not true for general stochastic control problems. In order to formulate the Verification Theorem properly we suppose that admissible portfolio strategies take values in a compact set $[-M, M], M \in \mathbb{R}_{+}$. We will later see that this assumption is no restriction for our applications. More precisely it holds

Theorem 1: Suppose $G \in C^{1,2}$ is a solution of the HJB-equation and $|G(t, x, i)| \leq K\left(1+|x|^{k}\right)$ for constants $K>0$ and $k \in \mathbb{N}$ and for all $i \in E$ and $0 \leq t \leq T$. Then

a) $G(t, x, i) \geq V(t, x, i)$ for all $0 \leq t \leq T, x \in \mathbb{R}_{+}$and $i \in E$.

b) If $\pi^{*}=\left(\pi_{t}^{*}\right)$ is a maximizer of the HJB-equation, i.e. $\pi_{s}^{*}$ maximizes

$$
u \mapsto \mathcal{G}^{u} G\left(s, X_{s}^{*}, Y_{s}\right)
$$

for all $s \in[t, T]$, where $X^{*}, \pi^{*}$ and $Y$ solve (3) then $G(t, x, i)=V(t, x, i)=V_{\pi^{*}}(t, x, i)$ for all $x \in \mathbb{R}_{+}, i \in E$. In particular, $\pi^{*}$ is an optimal portfolio strategy.

Proof: Let $\pi \in \mathcal{A}(t, \mathfrak{F})$ be an arbitrary portfolio strategy and $X^{\pi}$ the corresponding wealth process. Applying Ito's Lemma for semimartingales (see e.g. Jacod/Shiryaev (1987) Theorem 4.57) yields

$$
\begin{aligned}
& G\left(T, X_{T}^{\pi}, Y_{T}\right)= \\
& =G(t, x, i)+\int_{t}^{T}\left[G_{t}\left(s, X_{s}^{\pi}, Y_{s}\right)\right. \\
& +G_{x}\left(s, X_{s}^{\pi}, Y_{s}\right) X_{s}^{\pi}\left(r\left(Y_{s}\right)+\pi_{s}\left(\mu\left(Y_{s}\right)-r\left(Y_{s}\right)\right)\right) \\
& \left.+\frac{1}{2} G_{x x}\left(s, X_{s}^{\pi}, Y_{s}\right)\left(X_{s}^{\pi} \pi_{s} \sigma\left(Y_{s}\right)\right)^{2}\right] d s \\
& +\int_{t}^{T} G_{x}\left(s, X_{s}^{\pi}, Y_{s}\right) X_{s}^{\pi} \pi_{s} \sigma\left(Y_{s}\right) d W_{s} \\
& +\sum_{t \leq s<T}\left[G\left(s, X_{s}^{\pi}, Y_{s+}\right)-G\left(s, X_{s}^{\pi}, Y_{s}\right)\right]
\end{aligned}
$$

Let us denote by $q_{0}$ the jump measure of $Y$ and by $T_{n}$ the successive jump time points. Then

$$
q_{0}([0, t] \times\{j\})=\sum_{n \in \mathbb{N}} I_{\left[Y_{T_{n}+}=j, T_{n} \leq t\right]} .
$$

The compensator of $q_{0}$ is given by

$$
\nu([0, t] \times\{j\})=\int_{0}^{t} \sum_{i \neq j} q_{i j} I_{\left[Y_{s}=i\right]} d s .
$$

Hence, we have

$$
\begin{aligned}
& \sum_{t \leq s<T}\left[G\left(s, X_{s}^{\pi}, Y_{s+}\right)-G\left(s, X_{s}^{\pi}, Y_{s}\right)\right]= \\
& =\int_{t}^{T} \sum_{j \in E}\left[G\left(s, X_{s}^{\pi}, j\right)-G\left(s, X_{s}^{\pi}, Y_{s}\right)\right]\left(q_{0}-\nu\right)(d s, j) \\
& +\int_{t}^{T} \sum_{j \in E}\left[G\left(s, X_{s}^{\pi}, j\right)-G\left(s, X_{s}^{\pi}, Y_{s}\right)\right] q_{Y_{s} j} d s
\end{aligned}
$$


Since $G$ satisfies the HJB equation we obtain

$$
\begin{aligned}
& G\left(T, X_{T}^{\pi}, Y_{T}\right) \leq G(t, x, i) \\
& +\int_{t}^{T} G_{x}\left(s, X_{s}^{\pi}, Y_{s}\right) X_{s}^{\pi} \pi_{s} \sigma\left(Y_{s}\right) d W_{s} \\
& +\int_{t}^{T} \sum_{j \in E}\left[G\left(s, X_{s}^{\pi}, j\right)-G\left(s, X_{s}^{\pi}, Y_{s}\right)\right]\left(q_{0}-\nu\right)(d s, j)
\end{aligned}
$$

Next we take expectation on both sides. Note that $G\left(T, X_{T}^{\pi}, Y_{T}\right)=$ $U\left(X_{T}^{\pi}\right)$ due to the boundary condition. As in Fleming/Soner (1993) Theorem IV.3.1, it follows that the expectation of the first integral vanishes. Since

$$
E^{t, x, i}\left[\sum_{0<T_{i} \leq T}\left|G\left(T_{i}, X_{T_{i}}^{\pi}, Y_{T_{i}}\right)\right|<\infty\right.
$$

due to the growth condition on $G$, it follows with Theorem 26.12 in Davis (1993) that the second integral is a martingale and thus becomes zero under expectation. Hence, we obtain

$$
E^{t, x, i}\left[U\left(X_{T}^{\pi}\right)\right] \leq G(t, x, i) .
$$

Obviously if $\pi^{*}$ is a maximizer of the HJB equation we obtain equality. This observation concludes the proof.

In the following sections we solve the portfolio problem for a number of different utility functions.

\section{B. Portfolio-Optimization with Logarithmic Utility}

First we assume that the utility function is given by $U(x)=$ $\log (x)$. In this case it can be shown in a rather general setting that the optimal portfolio strategy invests a constant fraction $\pi^{*}(t, x, i)=$ $\frac{\mu(i)-r(i)}{\sigma^{2}(i)}$ of the wealth into the stock (see e.g. Goll/Kallsen (2000)). We will prove this result via stochastic control and give several representations of the value function which enable us in particular to compare the Markov-modulated investment problem to the situation with constant (average) volatility and constant (average) squared market price of risk.

Theorem 2: In the case of logarithmic utility the optimal portfolio strategy is given by

$$
\pi^{*}(t, x, i)=\frac{\mu(i)-r(i)}{\sigma^{2}(i)}
$$

and the optimal value is given by

$$
V(t, x, i)=\log (x)+g(t, i)
$$

where $g(t, i)$ is the unique solution of the following system of linear differential equations

$g_{t}(t, i)+r(i)+\frac{1}{2}\left(\frac{\mu(i)-r(i)}{\sigma(i)}\right)^{2}+\sum_{j \in E} q_{i j}[g(t, j)-g(t, i)]=0$

with boundary condition $g(T, i)=0$ for $i \in E$.

Proof: Suppose a solution $G$ of the HJB-equation can be written as $G(t, x, i)=\log (x)+g(t, i)$ with $g(\cdot, i) \in C^{1}$ for all $i \in E$. Hence, we obtain

$$
\begin{aligned}
G_{t} & =g_{t}(t, i) \\
G_{x} & =\frac{1}{x} \\
G_{x x} & =-\frac{1}{x^{2}} .
\end{aligned}
$$

Since the mapping $x \mapsto G(t, x, i)$ is concave, the maximizer $\pi^{*}$ of the HJB-equation is given by

$$
\pi^{*}(t, x, i)=-\frac{\mu(i)-r(i)}{\sigma^{2}(i)} \frac{G_{x}(t, x, i)}{x G_{x x}(t, x, i)} .
$$

Inserting the derivatives and $\pi^{*}$ in the HJB-equation leaves us with $g_{t}(t, i)+r(i)+\frac{1}{2}\left(\frac{\mu(i)-r(i)}{\sigma(i)}\right)^{2}+\sum_{j \in E} q_{i j}[g(t, j)-g(t, i)]=0$

and boundary condition $g(T, i)=0$ for $i \in E$. It is well-known that this system of differential equations has a unique solution $g$. As a result, our function $g$ satisfies the HJB equation, $G \in C^{1,2}$ and $|G(t, x, i)| \leq K(1+|x|)$ for a suitable constant $K$. Since $\pi^{*}$ is an admissible portfolio strategy, the result follows from the Verification Theorem 1 .

Remark 2: 1) In order to apply Theorem 1 we have to maximize over all portfolio strategies with values in $[-M, M]$. However, for large $M$ the fraction $\pi^{*}(t, x, i)=\frac{\mu(i)-r(i)}{\sigma^{2}(i)} \in$ $(-M, M)$. Thus, $\pi^{*}$ is also optimal when maximizing over all portfolio strategies. This observation is also valid for the CRRA utility.

2) In the model with known deterministic market coefficients $\left(r_{t}\right),\left(\mu_{t}\right),\left(\sigma_{t}\right)$ the solution of the portfolio problem is given by

$$
\pi^{*}(t, x)=\frac{\mu_{t}-r_{t}}{\sigma_{t}^{2}}
$$

and

$$
V(t, x)=\log (x)+\int_{t}^{T} r_{s}+\frac{1}{2}\left(\frac{\mu_{s}-r_{s}}{\sigma_{s}}\right)^{2} d s .
$$

In order to investigate the influence of the Markov-modulation on the value of the optimization problem, the following Feyman-Kac type representation of the value function is more convenient.

Lemma 1: The function $g(t, i)$ appearing in the value function of Theorem 2 can be written as

$$
g(t, i)=E^{t, i}\left[\int_{t}^{T} r\left(Y_{s}\right)+\frac{1}{2}\left(\frac{\mu\left(Y_{s}\right)-r\left(Y_{s}\right)}{\sigma\left(Y_{s}\right)}\right)^{2} d s\right] .
$$

Proof: Suppose $g(t, i)$ solves the system of linear differential equations given in Theorem 2. An application of Ito's Lemma for $t<s \leq T$ gives

$g\left(T, Y_{T}\right)=g(t, i)+\int_{t}^{T} g_{t}\left(s, Y_{s}\right) d s+\sum_{t \leq s<T}\left[g\left(s, Y_{s+}\right)-g\left(s, Y_{s}\right)\right]$.

Replacing $g_{t}\left(s, Y_{s}\right)$ by

$$
-r\left(Y_{s}\right)-\frac{1}{2}\left(\frac{\mu\left(Y_{s}\right)-r\left(Y_{s}\right)}{\sigma\left(Y_{s}\right)}\right)^{2}-\sum_{j \in E} q_{Y_{s} j}\left[g(s, j)-g\left(s, Y_{s}\right)\right]
$$

and taking expectation yields

$$
\begin{aligned}
& E^{t, i}\left[g\left(T, Y_{T}\right)\right]=g(t, i) \\
& -E^{t, i}\left[\int_{t}^{T} r\left(Y_{s}\right)+\frac{1}{2}\left(\frac{\mu\left(Y_{s}\right)-r\left(Y_{s}\right)}{\sigma\left(Y_{s}\right)}\right)^{2} d s\right] .
\end{aligned}
$$

Since $g\left(T, Y_{T}\right)=0$ the statement follows.

Suppose now that the Markov chain $Y$ has a unique stationary distribution $p=\left(p_{j}, j \in E\right)$. Let

$$
\bar{r}:=\sum_{j \in E} p_{j} r(j) \quad \text { and } \quad \bar{R}^{2}:=\sum_{j \in E} p_{j}\left(\frac{\mu(j)-r(j)}{\sigma(j)}\right)^{2}
$$

be the average interest rate and the average squared market price of risk. We want to compare the value function $\bar{V}(t, x)$ obtained in the model with averaged data $\bar{r}$ and $\bar{R}^{2}$ with the value function $E^{p}\left[V\left(t, x, Y_{0}\right)\right]$ in the Markov-modulated case with $Y_{0} \stackrel{d}{=} p$. It is easy to see that

$$
E^{p}\left[V\left(t, x, Y_{0}\right)\right]=\bar{V}(t, x) .
$$

This means that in the case of logarithmic utility it is sufficient to know the averaged data in order to compute the value of the portfolio problem. 


\section{Portfolio-Optimization with CRRA Utility}

Next we assume that the utility function is of Constant Relative Risk Aversion (CRRA), i.e. given by $U(x)=\frac{1}{\gamma} x^{\gamma}$ with $0<\gamma<1$. $1-\gamma$ is called risk aversion coefficient. This is another case where the HJB equation can be solved explicitly in the classical setting.

Theorem 3: In the case of CRRA utility the optimal portfolio strategy is given by

$$
\pi^{*}(t, x, i)=\frac{1}{1-\gamma} \cdot \frac{\mu(i)-r(i)}{\sigma^{2}(i)}
$$

and the optimal value is given by

$$
V(t, x, i)=\frac{1}{\gamma} x^{\gamma} \cdot g(t, i)
$$

where $g(t, i)$ is the unique solution of the following system of linear differential equations

$$
g_{t}(t, i)+a(i) g(t, i)+\sum_{j \in E} q_{i j}[g(t, j)-g(t, i)]=0
$$

with boundary condition $g(T, i)=1$ and

$$
a(i)=r(i) \gamma+\frac{1}{2} \frac{\gamma}{1-\gamma}\left(\frac{\mu(i)-r(i)}{\sigma(i)}\right)^{2}
$$

for $i \in E$.

Proof: Suppose a solution $G$ of the HJB equation can be written as $G(t, x, i)=\frac{1}{\gamma} x^{\gamma} g(t, i)$ with $g(\cdot, i) \in C^{1}$ for all $i \in E$. The derivatives are then given by

$$
\begin{aligned}
G_{t} & =\frac{1}{\gamma} x^{\gamma} g_{t}(t, i) \\
G_{x} & =x^{\gamma-1} g(t, i) \\
G_{x x} & =(\gamma-1) x^{\gamma-2} g(t, i) .
\end{aligned}
$$

If $g \geq 0$, the mapping $x \mapsto G(t, x, i)$ is concave and the maximizer $\pi^{*}$ of the HJB equation is given by

$$
\pi^{*}(t, x, i)=-\frac{\mu(i)-r(i)}{\sigma^{2}(i)} \frac{G_{x}(t, x, i)}{x G_{x x}(t, x, i)} .
$$

Inserting the derivatives and $\pi^{*}$ in the HJB equation gives

$$
\begin{aligned}
& \frac{1}{\gamma} g_{t}(t, i)+g(t, i)\left(r(i)+\frac{1}{2(1-\gamma)}\left(\frac{\mu(i)-r(i)}{\sigma(i)}\right)^{2}\right) \\
& +\frac{1}{\gamma} \sum_{j \in E} q_{i j}[g(t, j)-g(t, i)]=0
\end{aligned}
$$

and boundary condition $g(T, i)=1$ for $i \in E$. This differential equation has a unique positive solution $g$ (see Lemma 2 below for the fact that $g \geq 0)$. Therefore $G \in C^{1,2}$ and $|G(t, x, i)| \leq K(1+|x|)$ and solves the HJB equation. Since $\pi^{*}$ is an admissible portfolio strategy, the result follows from Theorem 1.

Remark 3: In the model with known deterministic market coefficients $\left(r_{t}\right),\left(\mu_{t}\right),\left(\sigma_{t}\right)$ the solution of the portfolio problem is given by

$$
\pi^{*}(t, x)=\frac{1}{1-\gamma} \cdot \frac{\mu_{t}-r_{t}}{\sigma_{t}^{2}}
$$

and

$$
V(t, x)=\frac{1}{\gamma} x^{\gamma} \cdot \exp \left\{\int_{t}^{T} \gamma r_{s}+\frac{1}{2} \frac{\gamma}{1-\gamma}\left(\frac{\mu_{s}-r_{s}}{\sigma_{s}}\right)^{2} d s\right\} .
$$

In order to investigate the influence of the Markov-modulation on the value of the investment problem, the following Feyman-Kac type representation of the value function is more convenient.
Lemma 2: The function $g(t, i)$ appearing in the value function of Theorem 3 can be written as

$$
g(t, i)=E^{t, i}\left[\exp \left\{\int_{t}^{T} a\left(Y_{s}\right) d s\right\}\right]
$$

where $a(\cdot)$ is defined in Theorem 3 .

Proof: Suppose first, $g(t, i)$ solves the system of differential equations (5). Using Ito's Lemma we obtain in the same way as in Lemma 1 that

$$
g(t, i)=1+E^{t, i}\left[\int_{t}^{T} g\left(s, Y_{s}\right) a\left(Y_{s}\right) d s\right] .
$$

Equation (6) has a unique solution $g(t, i)$. This can easily be proved by contradiction.

Now consider the function

$$
\tilde{g}(t, i)=E^{t, i}\left[\exp \left\{\int_{t}^{T} a\left(Y_{s}\right) d s\right\}\right] .
$$

It holds that

$$
\begin{aligned}
& 1+E^{t, i}\left[\int_{t}^{T} \tilde{g}\left(\tau, Y_{\tau}\right) a\left(Y_{\tau}\right) d \tau\right] \\
= & 1+E^{t, i}\left[\int_{t}^{T} E\left[\exp \left\{\int_{\tau}^{T} a\left(Y_{s}\right) d s\right\} \mid \mathcal{F}_{\tau}\right] a\left(Y_{\tau}\right) d \tau\right] \\
= & 1+E^{t, i}\left[\int_{t}^{T} \exp \left\{\int_{\tau}^{T} a\left(Y_{s}\right) d s\right\} a\left(Y_{\tau}\right) d \tau\right] \\
= & E^{t, i}\left[\exp \left\{\int_{t}^{T} a\left(Y_{s}\right) d s\right\}\right]=\tilde{g}(t, i) .
\end{aligned}
$$

Due to the uniqueness we have $g=\tilde{g}$ and the statement follows.

Suppose now that the Markov chain $Y$ has a unique stationary distribution $p=\left(p_{j}, j \in E\right)$ and let

$$
\bar{r}:=\sum_{j \in E} p_{j} r(j) \quad \text { and } \quad \bar{R}^{2}:=\sum_{j \in E} p_{j}\left(\frac{\mu(j)-r(j)}{\sigma(j)}\right)^{2}
$$

be the average interest rate and the average squared market price of risk. We want to compare the value function $\bar{V}(t, x)$ obtained in the model with averaged data $\bar{r}$ and $\bar{R}^{2}$ with the value function $E^{p}\left[V\left(t, x, Y_{0}\right)\right]$ in the Markov-modulated case with $Y_{0} \stackrel{d}{=} p$. Using Jensen's inequality

$$
\begin{aligned}
& E^{p}\left[V\left(t, x, Y_{0}\right)\right]=\frac{1}{\gamma} x^{\gamma} . \\
& E\left[\exp \left\{\int_{t}^{T} \gamma r\left(Y_{s}\right)+\frac{1}{2} \frac{\gamma}{1-\gamma}\left(\frac{\mu\left(Y_{s}\right)-r\left(Y_{s}\right)}{\sigma\left(Y_{s}\right)}\right)^{2} d s\right\}\right] \\
& \geq \frac{1}{\gamma} x^{\gamma} \cdot \exp \left\{(T-t) \bar{r} \gamma+\frac{1}{2} \frac{\gamma}{1-\gamma} \bar{R}^{2}(T-t)\right\}=\bar{V}(t, x)
\end{aligned}
$$

Thus, the expected utility in the Markov-modulated case is larger which means that an agent can take advantage of a changing volatility.

\section{Benchmark-Optimization}

We suppose now that the utility function is given by

$$
U(x)=\left\{\begin{array}{l}
1, \text { if } x \geq b \\
0, \text { if } x<b
\end{array}\right.
$$

for some fixed $b \in \mathbb{R}_{+}$. This means that we want to maximize the probability that our terminal wealth exceeds the goal $b$. Situations like this arise for example in the context of professional portfolio management where an agent's portfolio performance is solely measured by a certain benchmark. Mathematically this problem is more demanding. In his paper of 1999 , Browne has solved this problem 
for deterministic market date. The optimal (total) amount of money which has to be invested in the stock at time $t \in[0, T]$ is given by

$$
f_{t}^{*}=\frac{\sigma_{t}^{-1} \theta_{t}}{\sqrt{\int_{t}^{T} \theta_{s}^{2} d s}} b B(t, T) \phi\left(\Phi^{-1}\left(\frac{X_{t}^{*}}{b B(t, T)}\right)\right)
$$

where $B(t, T)=\exp \left\{-\int_{t}^{T} r_{s} d s\right\}, \theta_{t}=\sigma_{t}^{-1}\left(\mu_{t}-r_{t}\right)$ and $X^{*}=$ $\left(X_{t}^{*}\right)$ is the wealth process generated by $f^{*} . \Phi, \phi$ are the cumulative distribution function and density respectively of a standard normal variate. The corresponding value function reads

$$
V(t, x)=\Phi\left(\Phi^{-1}\left(\frac{x}{b B(t, T)}\right)+\sqrt{\int_{t}^{T} \theta_{s}^{2} d s}\right) .
$$

In the case of stochastic market data we are only partly able to solve this problem. We assume now that $r(\cdot) \equiv r$ and $\mu(\cdot) \equiv \mu$, i.e. only volatility is Markov-modulated. Note that in contrast to the previous sections $f_{t}$ is now the total amount of money invested in the stock at time $t$, i.e. we have the following relation $f_{t}=\pi_{t} X_{t}^{f} \cdot\left(X_{t}^{f}-f_{t}\right)$ is then the amount of money invested in the bond at time $t$. Let us first consider the following special investment strategy $\hat{f}$ :

$$
\hat{f}_{t}=\frac{1}{\sigma\left(Y_{t}\right) \sqrt{T-t}} b B(t, T) \phi\left(\Phi^{-1}\left(\frac{\hat{X}_{t}}{b B(t, T)}\right)\right) .
$$

where $\hat{X}=\left(\hat{X}_{t}\right)$ is the wealth process under investment strategy $\hat{f}$.

Theorem 4: The wealth process under investment strategy $\hat{f}$ defined in (7) is for $0 \leq t<T$ given by

$$
\hat{X}_{t}=b B(t, T) \Phi\left(\frac{W_{t}+\int_{0}^{t} \frac{\mu-r}{\sigma\left(Y_{s}\right)} d s+\sqrt{T} \Phi^{-1}\left(\frac{x}{b B(0, T)}\right)}{\sqrt{T-t}}\right) .
$$

The corresponding terminal wealth is given by

$$
\begin{aligned}
& E^{t, x, i}\left[U\left(\hat{X}_{T}\right)\right]= \\
& \Phi\left(\Phi^{-1}\left(\frac{x}{b B(t, T)}\right)+\frac{1}{\sqrt{T-t}} \int_{t}^{T} \frac{\mu-r}{\sigma\left(Y_{s}\right)} d s\right) .
\end{aligned}
$$

Proof: Using Ito's Lemma we show that $\hat{X}=\left(\hat{X}_{t}\right)$ defined in (8) satisfies the following stochastic differential equation for the wealth process

$$
\begin{aligned}
d \hat{X}_{t} & =\left[r \hat{X}_{t}+\hat{f}_{t}(\mu-r)\right] d t+\hat{f}_{t} \sigma\left(Y_{t}\right) d W_{t} \\
\hat{X}_{0} & =x
\end{aligned}
$$

on $[0, T)$. Since the solution of the stochastic differential equation is unique, the representation of $\hat{X}$ follows. In order to simplify things slightly we define

$$
\hat{V}_{t}:=\hat{X}_{t}(B(t, T) b)^{-1} .
$$

Using the product rule it is easy to see that it suffices to verify that $\hat{V}=\left(\hat{V}_{t}\right)$ solves the stochastic differential equation

$$
\begin{aligned}
d \hat{V}_{t}= & (\mu-r)(B(t, T) b)^{-1} \hat{f}_{t} d t+(B(t, T) b)^{-1} \hat{f}_{t} \sigma\left(Y_{t}\right) d W_{t} \\
= & \frac{\mu-r}{\sigma\left(Y_{t}\right) \sqrt{T-t}} \phi\left(\Phi^{-1}\left(\hat{V}_{t}\right)\right) d t \\
& +\frac{1}{\sqrt{T-t}} \phi\left(\Phi^{-1}\left(\hat{V}_{t}\right)\right) d W_{t} \\
\hat{V}_{0}= & \frac{x}{b B(0, T)}
\end{aligned}
$$

Denote now $\theta_{s}=\frac{\mu-r}{\sigma\left(Y_{s}\right)}$. An application of Ito's Lemma to the Brownian motion $W=\left(W_{t}\right)$, to the process $Z=\left(Z_{t}:=\int_{0}^{t} \theta_{s} d s\right)$ and to the function

$$
g(w, z, t):=\Phi\left(\left(w+z+\sqrt{T} \Phi^{-1}\left(\hat{V}_{0}\right)\right)(T-t)^{-\frac{1}{2}}\right)
$$

gives

$$
d \hat{V}_{t}=g_{t} d t+g_{w} d W_{t}+g_{z} \theta_{t} d t+\frac{1}{2} g_{w w} d t .
$$

Inserting the derivatives implies (9) for $t \in[0, T)$. Since $\hat{V}_{t}$ is continuous and bounded on $[0, T]$ we have $\lim _{t \rightarrow T} \hat{V}_{t}=\hat{V}_{T}$ and thus

$$
\begin{aligned}
& P^{t, x, i}\left(\hat{X}_{T} \geq b\right)=P^{t, x, i}\left(\lim _{t \rightarrow T} \hat{X}_{t} \geq b\right) \\
= & P^{t, x, i}\left(W_{T-t}+\int_{t}^{T} \theta_{s} d s+\sqrt{T-t} \Phi^{-1}\left(\frac{x}{b B(t, T)}\right) \geq 0\right) \\
= & P^{t, x, i}\left(-\frac{W_{T-t}}{\sqrt{T-t}} \leq \frac{1}{\sqrt{T-t}} \int_{t}^{T} \theta_{s} d s+\Phi^{-1}\left(\frac{x}{b B(t, T)}\right)\right)
\end{aligned}
$$

yields the result.

Theorem 5: If $\mu=r$, the investment strategy $\hat{f}$ given in (7) is optimal for the benchmark optimization problem.

Proof: Let $f$ be an arbitrary investment strategy. For $\mu=r$ the process $V_{t}^{f}=X_{t}^{f}(B(t, T) b)^{-1}$ for $0 \leq t<T$ is given by

$$
V_{t}^{f}=V_{0}^{f}+\int_{0}^{t}(B(s, T) b)^{-1} f_{s} \sigma\left(Y_{s}\right) d W_{s} .
$$

Since $\left(V_{t}^{f}\right)$ is bounded, $\left(V_{t}^{f}\right)$ is a martingale for any portfolio strategy $f$. Thus, we obtain with the Tchebychev inequality

$$
P^{0, x, i}\left(X_{T}^{f} \geq b\right)=P^{0, x, i}\left(V_{T}^{f} \geq 1\right) \leq \frac{x}{b B(0, T)}
$$

and therefore

$$
V(0, x, i) \leq \frac{x}{b B(0, T)} .
$$

On the other hand, Theorem 4 shows that the upper bound is achieved for $\mu=r$ under policy $\hat{f}$. This observation completes the proof.

Now suppose the intensity matrix $Q$ of the Markov chain $Y$ is multiplied by a constant $c>0$. In this model we index all appearing processes with $c$. We will investigate the two cases where $c \rightarrow \infty$ which means that the volatility changes rapidly and $c \rightarrow 0$ which means that the volatility remains the same for a long time. Once again let us assume that $p=\left(p_{j}, j \in E\right)$ is the unique stationary distribution of $Y$. In these cases it is well-known that

$$
\begin{gathered}
\int_{0}^{t} \frac{1}{\sigma\left(Y_{s}^{c}\right)} d s \Rightarrow \frac{1}{\bar{\sigma}} t \text { for } c \rightarrow \infty \\
\int_{0}^{t} \frac{1}{\sigma\left(Y_{s}^{c}\right)} d s \Rightarrow \frac{1}{\sigma\left(y_{0}\right)} t \text { for } c \rightarrow 0
\end{gathered}
$$

where $\bar{\sigma}^{-1}=\sum_{i \in E} p_{i} \sigma^{-1}(i), y_{0}$ is the initial state $Y_{0}=y_{0}$ and $\Rightarrow$ denotes the usual weak convergence. Let us denote by

$$
V_{\sigma}(t, x)=\Phi\left(\Phi^{-1}\left(\frac{x}{b B(t, T)}\right)+\sqrt{T-t} \frac{\mu-r}{\sigma}\right)
$$

the value function of the benchmark optimization problem with constant volatility $\sigma$. Then we obtain with the properties of weak convergence:

Corollary 1: For $c \rightarrow \infty$ and $c \rightarrow 0$, the investment strategy $\hat{f}$ defined in (7) is asymptotically optimal in the sense that

$$
\begin{gathered}
E^{0, x, i}\left[U\left(\hat{X}_{T}^{c}\right)\right] \rightarrow V_{\bar{\sigma}}(0, x) \text { for } c \rightarrow \infty \\
E^{0, x, i}\left[U\left(\hat{X}_{T}^{c}\right)\right] \rightarrow V_{\sigma\left(y_{0}\right)}(0, x) \text { for } c \rightarrow 0
\end{gathered}
$$

Remark 4: It follows from the results of Kulldorff (1993) that the benchmark optimization problem is equivalent to maximizing the terminal utility

$$
U(x)=\left\{\begin{array}{l}
x, \text { if } x \leq b \\
b, \text { if } x \geq b
\end{array}\right.
$$

This seems to be a simpler function since it is in particular continuous. However, we were not able to exploit this fact for our analysis. 


\section{CONCLUSION}

Portfolio optimization with stochastic market data is more realistic than standard models with constant coefficients. The formulation of the market condition as a continuous-time Markov chain makes the analysis simpler as in the case of a driving diffusion. For the utility functions treated here, the maximal portfolio value can be computed as a solution of a simple linear differential equation. More complicated is the case of benchmark optimization. It remains open whether a closed form solution can be derived in the general Markovmodulated case.

\section{REFERENCES}

[1] S. Browne, "Reaching goals by deadline: digital options and continuoustime active portfolio management," Adv. Appl. Probab., vol. 31, pp. 551577, 1999.

[2] M. H. A. Davis, Markov models and optimization, Chapman \& Hall, London, 1993.

[3] G. B. Di Masi, Y. M. Kabanov and W. J. Runggaldier, ”Mean-variance hedging of options on stocks with Markov volatilities," Theory Probab. Appl., vol. 39, pp. 211-222, 1994.

[4] W. H. Fleming and D. Hernández-Hernández, " An optimal consumption model with stochastic volatility, "Finance \& Stochastics, vol. 7, pp. 245262, 2003.

[5] W. H. Fleming and R. W. Rishel, Deterministic and stochastic optimal control, Springer-Verlag, New York, 1975.

[6] W. H. Fleming and H. M. Soner, Controlled Markov processes and viscosity solutions, Springer-Verlag, New York, 1993.

[7] H. Föllmer and P. Leukert, "Quantile hedging.", Finance \& Stochastics, vol. 3, pp. 251-273, 1999.

[8] J. P. Fouque, G. Papanicolaou and K. R. Sircar, Derivatives in financial markest with stochastic volatility. Cambridge University Press, 2000.

[9] R. Frey and W. J. Runggaldier, "A nonlinear filtering approach to volatility estimation with a view towards high frequency data,' International Journal of Theoretical and Applied Finance, vol. 4, pp. 199-210, 2001.

[10] T. Goll and J. Kallsen, "Optimal portfolios for logarithmic utility," Stoch. Proc. Appl., vol. 89, pp. 31-48, 2000.

[11] S. Heston "A closed-form solution for options with stochastic volatility with applications to bond and currency options," Rev. of Financial Studies, vol. 6, pp. 327-343, 1993.

[12] T. S. Y. Ho and S. B. Lee, "Term structure movements and pricing interest contingent claims," J. Finance, vol. 41, pp. 1011-1029, 1986.

[13] J. Jacod and A. N. Shiryaev, Limit theorems for stochastic processes. Springer-Verlag, New York, 1987.

[14] R. Korn and H. Kraft, "A stochastic control approach to portfolio problems with stochastic interest rates," SIAM J. Contr. Optim., vol. 40, pp. 1250-1269, 2001.

[15] M. Kulldorff, "Optimal control of favorable games with a time limit," SIAM J. Contr. Optim., vol. 31, pp. 52-69, 1993.

[16] H. J. Kushner and P. G. Dupuis, Numerical methods for stochastic control problems in continuous time. Springer-Verlag, New York, 1992.

[17] A. Lioui and P. Poncet, "On optimal portfolio choice under stochastic interest rates," J. Econom. Dynam. Control, vol. 25, pp. 1841-1865, 2001.

[18] R. C. Merton, "Optimum consumption and portfolio rules in a continuous-time model," J. Econo. Theo., vol. 3, pp. 373-413, 1971; erratum, J. Econo. Theo., vol. 6, pp. 213-214, 1973.

[19] H. Pham and M. C. Quenez, "Optimal portfolio in partially observed stochastic volatility models," Ann. Appl. Probab., vol. 11, pp. 210-238, 2001

[20] O. Vasicek, "An equilibrium characterization of the term structure," $J$. of Financial Economics, vol. 5, pp. 177-188, 1977.

[21] T. Zariphopoulou, "Optimal investment and consumption models with non-linear stock dynamics," Mathem. Methods Operat. Res., vol. 50, pp. 271-296, 1999.

[22] T. Zariphopoulou, "A solution approach to valuation with unhedgeable risks," Finance \& Stochastics, vol. 5, pp. 61-82, 2001.

[23] Q. Zhang, "Stock trading: an optimal selling rule," SIAM J. Control Optim., vol. 40, pp. 64-87, 2001. 